$>$ A l'ère de la médecine personnalisée, la recherche de nouveaux marqueurs capables d'améliorer le diagnostic des patients devient primordiale. La notion de test diagnostic « compagnon», capable de guider les médecins et d'orienter les choix thérapeutiques, commence à voir le jour sous le concept de théranostic. Depuis leur découverte et l'observation d'étroites corrélations avec un contexte pathologique, les microARN (miARN) contenus dans les fluides biologiques représentent de nouvelles pistes prometteuses pour la mise au point de biomarqueurs. D'abord étudiés dans les cancers, leur champ d'application est immense et touche tous les aspects de la médecine. Cet article passe en revue les récentes avancées sur les miARN circulants, leur origine, leur fonction, mais aussi leur potentiel d'application en clinique. <

La recherche de biomarqueurs fiables, sensibles et peu invasifs est un enjeu de premier ordre pour la médecine moderne. En effet, ces biomarqueurs ont le potentiel de révolutionner la prise en charge médicale en améliorant le dépistage et le diagnostic, mais aussi en permettant de mieux appréhender la réponse des patients à un traitement donné. Les besoins en biomarqueurs pertinents sont très pressants, notamment en oncologie où un dépistage précoce est souvent associé à un meilleur pronostic pour les patients. Chaque domaine de la médecine est concerné, des maladies cardiovasculaires aux maladies auto-immunes en passant par les maladies neurodégénératives de plus en plus fréquentes dans nos populations. En plus de sa pertinence biologique, un biomarqueur se doit d'être facilement accessible et dosable, c'est pour cela que les fluides corporels (sérum, urine, etc.) représentent un matériel de choix pour ce type d'investigations. Depuis déjà plusieurs décennies, l'existence d'acides nucléiques libres dans la circulation a été décrite et envisagée comme outil diagnostique, mais depuis la découverte

\section{Les microARN circulants, \\ une nouvelle classe de biomarqueurs pour la médecine}

Sylvain Baulande, Audrey Criqui, Mathilde Duthieuw

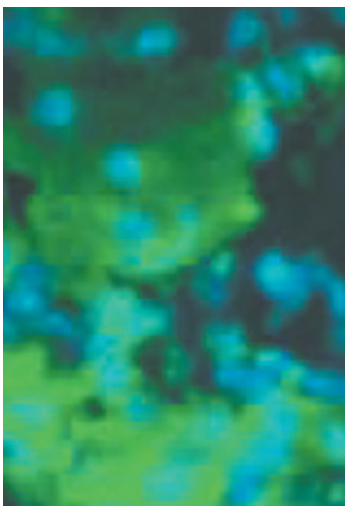

PartnerChip CEA, Génopole Campus 2, bâtiment G2, 2, rue Gaston Crémieux, 91000 Évry,

France.

sylvain.baulande@cea.fr

des microARN (miARN) chez l'homme en 2000 et leur détection dans la circulation en 2008, la caractérisation des miARN circulants est en plein essor (Figure 1) [1, 2]. Nombres d'études récentes suggèrent un fort potentiel prédictif à ces molécules qui pourraient bien constituer une nouvelle catégorie de biomarqueurs très utile en clinique.

\section{Les miARN, régulateurs post-transcriptionnels de l'expression des gènes}

Les découvertes issues du séquençage du génome humain ont permis de constater que seulement 1 à $2 \%$ de sa séquence codent pour des protéines. Alors que le reste du génome était, il y a peu, encore considéré comme dépourvu de fonction, il s'avère finalement que près de $90 \%$ de cette séquence est elle aussi transcrite. Les ARN non codants (ARNnc) qui en résultent sont vraisemblablement impliqués dans des mécanismes sophistiqués et encore peu connus visant à réguler l'expression des gènes $[3,4]$. Le mode d'action d'un sous-type d'ARNnc, appelés microARN (miARN), a été l'un des premiers à avoir été mis à jour $[5,6]$. Ces petits ARN intracellulaires longs de 22 nucléotides sont capables d'induire l'extinction (silencing) de l'expression de gènes par des mécanismes de régulations post-transcriptionnelles, en se fixant de manière ciblée aux parties 3' non traduites (3'UTR) des ARNm et en provoquant un blocage de la traduction ou la dégradation de ceux-ci (Figure 2) [7, 8]. Ce mécanisme n'est cependant pas exclusif; la fixation de miARN sur les régions 5'UTR est aussi possible et induit alors, soit une activation, soit une répression de la traduction [9]. Chez l'homme, 2578 miARN matures sont répertoriés dans la dernière version de la miRBase (Release20, http://www.mirbase.org/) [10], et plus de $60 \%$ des gènes 


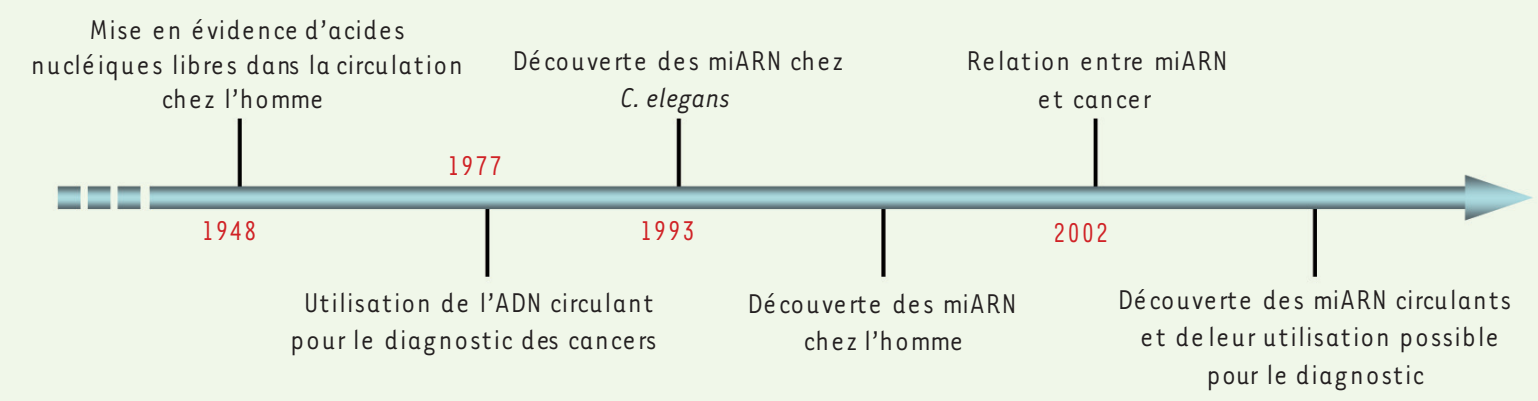

Figure 1. Dates clés des découvertes ayant permis la mise en évidence du pouvoir prédictif des miARN circulants en médecine.

sont susceptibles d'être régulés par les miARN [11]. Ce mécanisme fondamental de contrôle de l'expression des gènes est l'objet de perturbations importantes dans un contexte pathologique où des dérégulations de l'expression de ces miARN sont fréquemment observées [12].

\section{Sécrétion dans le milieu extracellulaire}

La présence de miARN dans le milieu extracellulaire a été mise en évidence en 2008 par Gilad et al. [2]. Cette découverte a fait polémique un temps, car beaucoup pensaient que ces miARN circulants n'étaient que la conséquence passive de la libération du contenu cytoplasmique lors de la mort cellulaire. On sait à présent que les cellules sont aussi capables d'une sécrétion complexe et finement régulée de ces miARN. Les miARN circulants sont principalement retrouvés sous la forme de quatre types de structures différentes associés ou non à des complexes lipidiques (Figure 3 ).

- Les exosomes (40-100 nm) tout d'abord, sont des microvésicules lipidiques formées à partir d'un processus d'invagination de la membrane plasmique donnant naissance à des endosomes qui se chargent alors en vésicules; celles-ci seront finalement libérées dans le milieu extracellulaire par fusion avec la membrane plasmique. Le contenu de ces vésicules exosomales, étroitement contrôlé par la cellule, se compose de divers types de molécules, comme des ARN messagers et des protéines, mais aussi des miARN.

- D’autres microvésicules, plus grandes que les exosomes $(0,1-1 \mu \mathrm{m})$ et issues du bourgeonnement de la membrane plasmique, sont aussi impliquées dans le transport extracellulaire des miARN. La composition de ces microvésicules en miARN est très similaire à celle de la cellule mère et ne semble pas être sujet à des mécanismes de sélection, comme c'est le cas pour les exosomes. De nombreux miARN sont sécrétés dans le milieu extracellulaire en association avec des microvésicules, comme miR-150 produit par les monocytes [13], ou encore miR-143 et miR-145 produits par des cellules endothéliales [14]. La caractérisation du contenu de ces microvésicules fait l'objet de nombreuses études dont les résultats ont été mis à jour dans la base de données ExoCarta [15] (http://www.exocarta.org/).

- Les miARN peuvent aussi être véhiculés par l'intermédiaire de corps apoptotiques $(0,5-2 \mu \mathrm{m})$ qui se forment lors des stades précoces de l'apoptose. Les cellules endothéliales, par exemple, relarguent le miR-126 dans la circulation par ce phénomène [16].

- Enfin, les lipoprotéines (HDL [high density lipoprotein] et LDL [low density lipoprotein]) sont elles aussi capables de transporter des miARN, les plus abondants dans les HDL étant miR-135a-1, miR-188 et miR-877 [17]. Cependant, la proportion des miARN circulants qui ne sont pas associés à des structures lipidiques, mais seulement complexés aux protéines argonaute (Ago2 le plus souvent), est de l'ordre de 90-95\% [18]. La très grande stabilité de ce complexe, qui protège les miARN des nombreuses RNases présentes dans la circulation, est probablement responsable de cette proportion élevée, qui ne serait que le reflet de la libération passive dans le milieu extracellulaire du contenu cytoplasmique lors de la mort cellulaire [19]. II existe, là encore, une base de données des miARN circulants appelée miRandola [20], dont l'objectif est de recenser et de documenter ces miARN extracellulaires (http://atlas.dmi.unict.it/mirandola/).

\section{Accumulation dans les fluides biologiques}

La présence de miARN dans les fluides biologiques a fait l'objet d'une caractérisation très détaillée dans une récente étude [21]. Les 12 types de fluides testés se sont tous avérés contenir des quantités significatives de miARN (Tableau 1). Les fluides comportant le moins de miARN différents sont l'urine, le liquide céphalo-rachidien et le liquide pleural, alors que la salive, le liquide séminal et le lait maternel semblent dotés d'une très grande diversité de molécules. Certaines d'entre elles sont abondantes et détectées dans la plupart des fluides, alors que d'autres ont une distribution plus spécifique (Tableau I). Les cellules responsables de la production de ces miARN sécrétés restent difficiles à déterminer. Dans le plasma, la proximité des cellules sanguines incite à les impliquer 


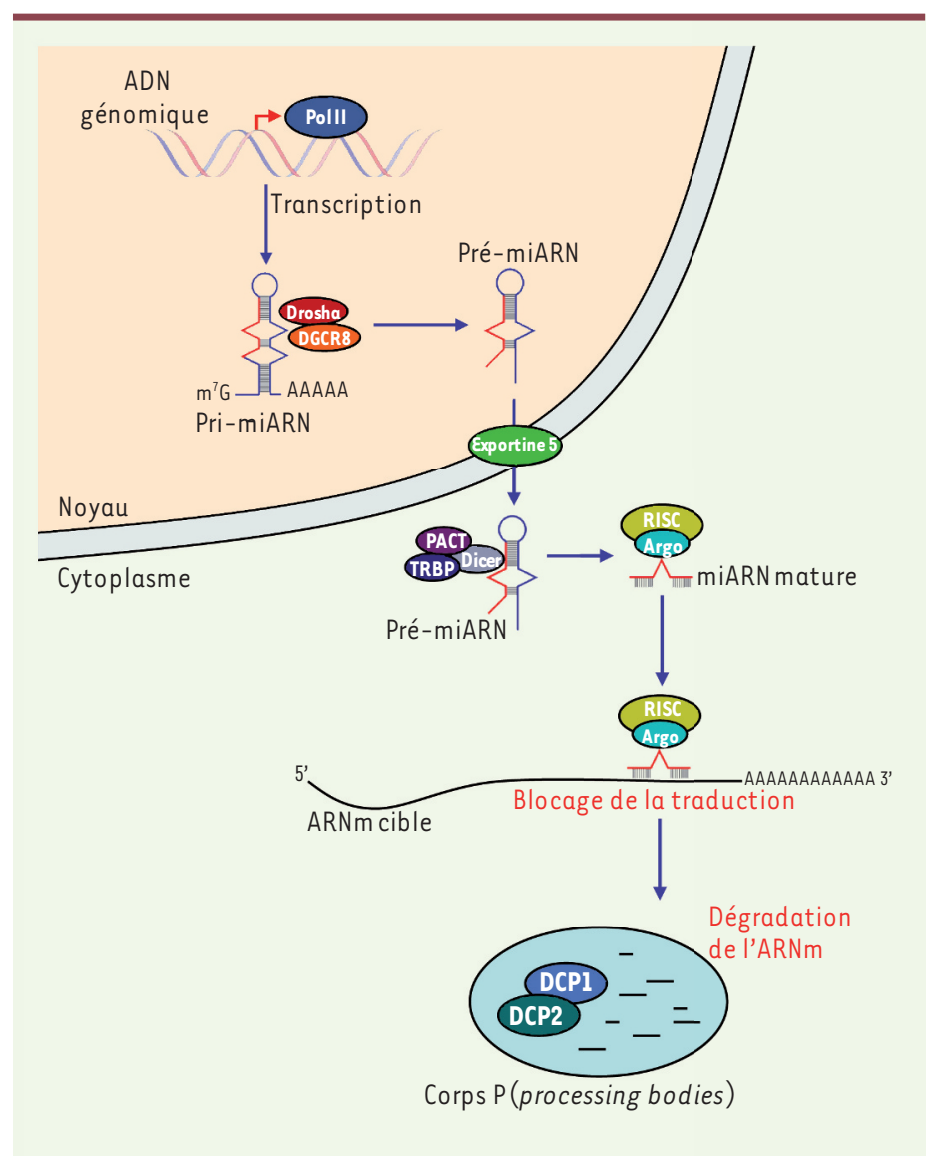

Figure 2. Régulation post-transcriptionnelle de l'expression des gènes par les miARN. Les gènes codant pour les miARN sont transcrits par l'ARN polymérase II (Pol II) en molécules de 60 à 100 nucléotides de long dotées d'une structure secondaire en tige/boucle appelées miARN primaires (pri-miARN). Dans le noyau, ces pri-miARN sont clivés par le complexe Drosha/DGCR8 (DiGeorge syndrome critical region-8) en pré-miARN, puis exportés vers le cytoplasme par l'intermédiaire de l'exportine 5 . Une fois sortis du noyau, les pré-miARN sont à nouveau clivés par le complexe DICER de manière à former les miARN matures (environ 22 nucléotides) pris en charge par des protéines de la famille des argonautes (Ago, de 1 à 4 chez l'homme) et intégrés au sein d'un complexe protéique appelé le RISC (RNA-induced silencing complex). Une fois assemblé, ce complexe peut se fixer spécifiquement à certaines séquences situées dans les parties 3' non traduites d'ARNm (3'UTR). La séquence en position 2-7 des miARN (seed) est responsable de l'adressage du complexe à la séquence cible. Si une homologie parfaite est responsable du clivage de l'ARNm, l'homologie partielle, majoritaire chez l'homme, conduit quant à elle à un blocage de la traduction suivi d'une dégradation des ARNm au sein des corps $P$ ( $P$-bodies), contenant notamment le complexe enzymatique DCP1 (decapping complex 1)/DCP2 responsable de la dégradation de la coiffe. TRBP : TAR-RNA binding protein.

dans cette production, ce d'autant plus qu'elles expriment la plupart des miARN observés dans la circulation. Cependant, la présence de miARN spécifiques de certains tissus, comme le foie (miR-122), les muscles (miR-133a) ou le cerveau (miR-124), plaide pour une origine diverse et complexe de ces molécules circulantes [22].

\section{Des régulateurs paracrines}

À ce jour, aucun effet fonctionnel n'a pu être mis en évidence pour les miARN complexés aux protéines argonaute, qui pourtant représentent la structure la plus abondante dans la circulation. Concernant les miARN associés à des structures lipidiques, leur captation par des cellules réceptrices est possible via des mécanismes d'endocytose, de fusion ou de phagocytose ; cependant, leur faible concentration dans la circulation générale rend peu vraisemblable un quelconque effet physiologique des miARN dans ces cellules [19].

Récemment, plusieurs travaux mettent en lumière les effets paracrines de miARN sécrétés dans des exosomes et qui, après leur recapture par des cellules hôtes présentes dans le micro-environnement, vont y inhiber l'expression de gènes cibles [13, 23]. Ainsi, l'induction de miR-143 et miR-145 par les cellules endothéliales en réponse aux contraintes de cisaillement (shear stress) est suivie d'une sécrétion de ces miR dans les exosomes, puis de leur captation par les cellules musculaires lisses dans lesquelles ils vont bloquer l'expression d'ARNm cibles $[14,24]$. Ce phénomène a même une importance fonctionnelle majeure, puisque les auteurs ont aussi démontré que ce mécanisme de communication extracellulaire, impliquant ces deux miARN, est capable de limiter le développement de plaques d'athérome chez des souris $A p o e^{-/-}[14,24]$. Ce type de régulation est aussi mis à contribution lors du développement tumoral : certaines cellules tumorales régulent, via des exosomes, l'expression des gènes de cellules avoisinantes afin d'induire un micro-environnement favorable à leur prolifération et à leur invasion. Une étude comparative de la composition en miARN de vésicules produites par des cellules de carcinomes hépatocellulaires a montré qu'elle était très différente de celle des cellules d'origine. Certains miARN comme miR-584, miR-517c ou bien miR-378, sont très fortement représentés dans ces exosomes, alors que leur expression cellulaire est à peine détectable [25]. Après la captation de ces vésicules par les cellules voisines, les miARN exogènes agissent sur les messagers cellulaires et bloquent l'expression de gènes de la voie TAKl (transforming growth factor $\beta$ activated kinase-1), connue pour être inactivée lors de la carcinogenèse hépatique [25]. De plus, certains miARN produits par des cellules cancéreuses régulent directement des récepteurs au profit de la croissance tumorale. C'est le cas de miR-21 et miR-29a, capables de se fixer directement au récepteur TLR8 (Toll-like receptor 8 ) humain en agissant comme un agoniste paracrine, induisant une réponse inflammatoire, prométastatique, des cellules immunitaires [26]. 


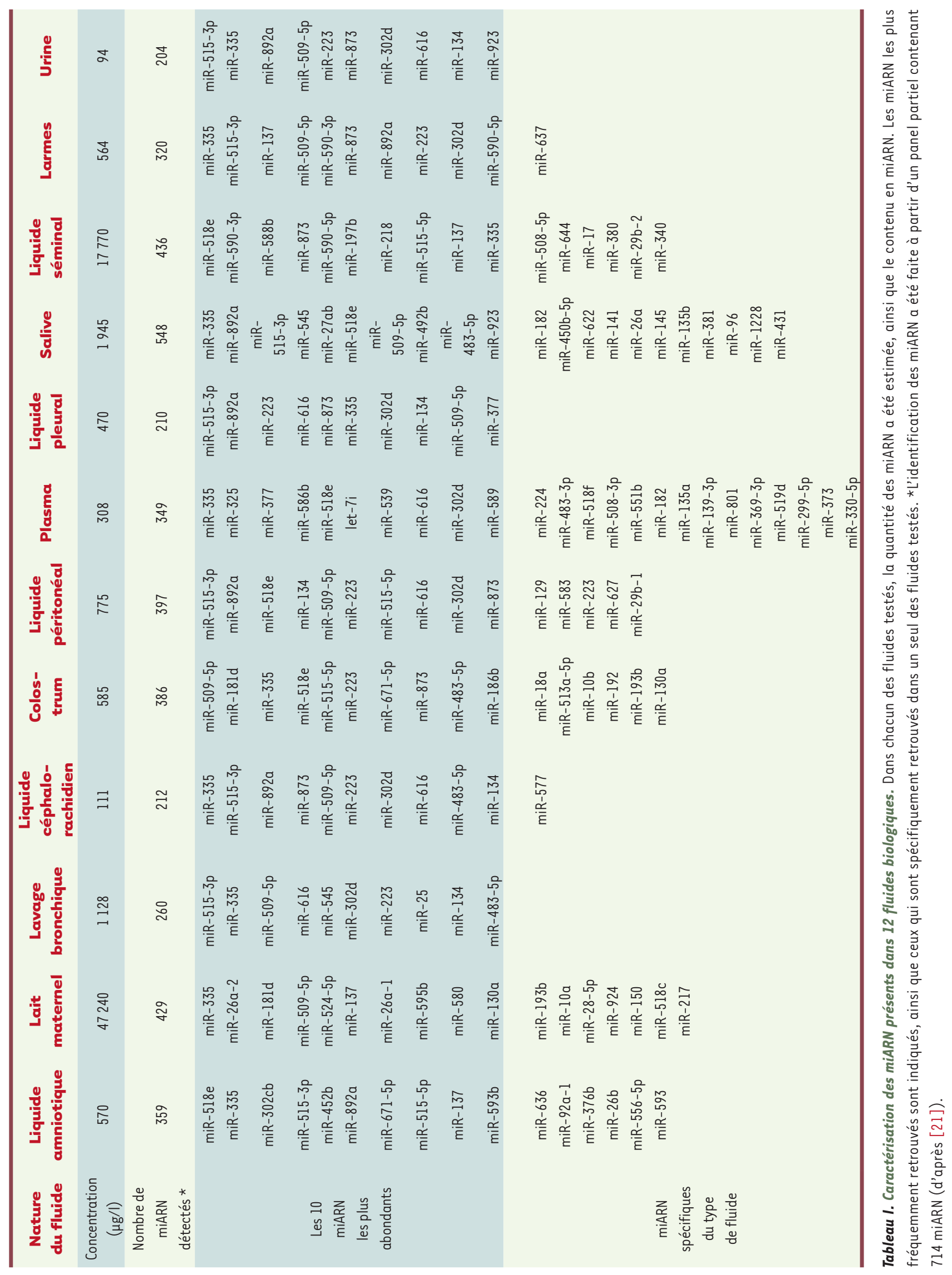




\section{Espace extracellulaire}

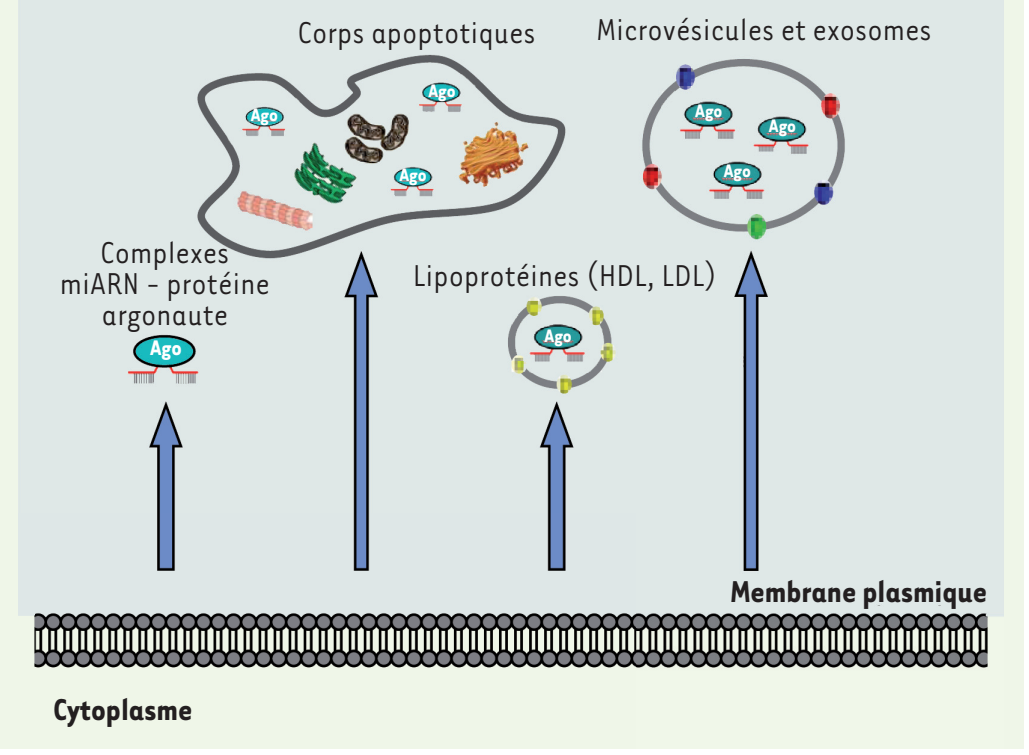

Figure 3. Exports des miARN dans le milieu extracellulaire. Les miARN présents dans l'espace extracellulaire sont produits par les cellules sous quatre formes principales. Tout d'abord, sous une forme non lipidique où les miARN sont juste complexés aux protéines argonaute qui les protègent de la dégradation. C'est la forme majoritaire que l'on retrouve dans le milieu extracellulaire. Dans les autres types d'export, les miARN sont associés à des structures lipidiques. Les corps apoptotiques qui résultent de la mort cellulaire par apoptose enferment dans ces structures des miARN cytoplasmiques qui se retrouvent ainsi dans la circulation. Les vésicules de sécrétion, ainsi que les exosomes produits par les cellules, sont aussi des mécanismes importants permettant la libération des miARN. Enfin, la présence de miARN associés aux lipoprotéines de type HDL a aussi été décrite et représente une autre voie d'export des miARN cellulaires.

\section{miARN circulants et pathologie humaine}

L'apparition d'un contexte pathologique est souvent associée à une perturbation importante de l'expression des miARN cellulaires dans les tissus concernés. La recherche de modifications quantitatives des miARN dans la circulation lors de ces situations pathologiques constitue donc un axe de recherche prometteur. La première démonstration d'un lien entre un miARN circulant et un cancer date de 2008. Une étude du sérum de 60 patients atteints de lymphomes diffus à grandes cellules $B(D L B C L)$ avait rapporté une augmentation significative des miARN miR-155, miR-21 et miR-210 par comparaison à une population contrôle [27]. Depuis, plusieurs centaines de publications ont décrit des modifications quantitatives de certains miARN présents dans les fluides biologiques (principalement le sérum) de patients par rapport à une cohorte contrôle (Tableau II). Ces observations ne se limitent pas aux cancers car de nombreuses pathologies sont concernées comme les maladies cardiovasculaires, le diabète de type 2, les affections hépatiques ou encore les infections virales ou le sepsis [28].

Deux mécanismes distincts rendent compte de ces modifications quantitatives. Elles peuvent être consécutives à un relargage passif lié à une lyse tissulaire provoquée par la maladie, comme par exemple lors d'un infarctus du myocarde. Les miARN miR-208, miR-499 et miR-133, dont l'expression est restreinte au tissu musculaire, sont ainsi libérés dans la circulation suivant le degré d'atteinte du muscle cardiaque [29]. De la même manière, la concentration sérique de miR-122 augmente proportionnellement à la sévérité d'une hépatite $C$ chronique, mais aussi lors d'une stéatose hépatique non alcoolique (NASH) [30]. Ces deux exemples illustrent l'intérêt du suivi du taux de ces miARN spécifiques de tissus pour apprécier une atteinte tissulaire. Dans ce type de situation, l'augmentation de certains miARN dans le sérum n'est donc qu'une conséquence indirecte de la pathologie, alors que dans d'autres cas, les miARN pourraient avoir un rôle pathogénique et participer à l'évolution de la maladie. Dans le diabète de type 2 par exemple, une modification des quantités sériques de plusieurs miARN, dont miR-126, miR-223 et miR-15a, est détectée avant même le développement de la maladie, ce qui suggère fortement I'implication directe de ces miARN dans la mise en place de la physiopathologie diabétique [31, 38].

\section{Une nouvelle classe de biomarqueurs}

L'association étroite entre contextes pathologiques et taux de certains miARN dans la circulation fait de ces derniers d'excellents candidats biomarqueurs pour la clinique, et les études se multiplient depuis 2008 pour valider de telles associations.

Améliorer le dépistage précoce des cancers est primordial afin de permettre une prise en charge rapide et d'optimiser les chances de guérison. La prise en charge du cancer du poumon par exemple, qui est le cancer dont la mortalité est la plus importante, pourrait être grandement améliorée si des biomarqueurs pertinents étaient à la disposition des médecins. La société Rosetta Genomics propose déjà des tests diagnostiques basés sur l'expression des miARN. Le test miRview lung par exemple, utilise une signature basée sur huit miARN qui permet de différencier les quatre principales classes de cancer du poumon à partir d'une biopsie avec une précision de $94 \%$ [32]. Pour les miARN circulants, outil diagnostique précoce non invasif de choix, des travaux récents ont identifié un panel de dix miARN capable de prédire le développement d'un cancer du poumon de type 


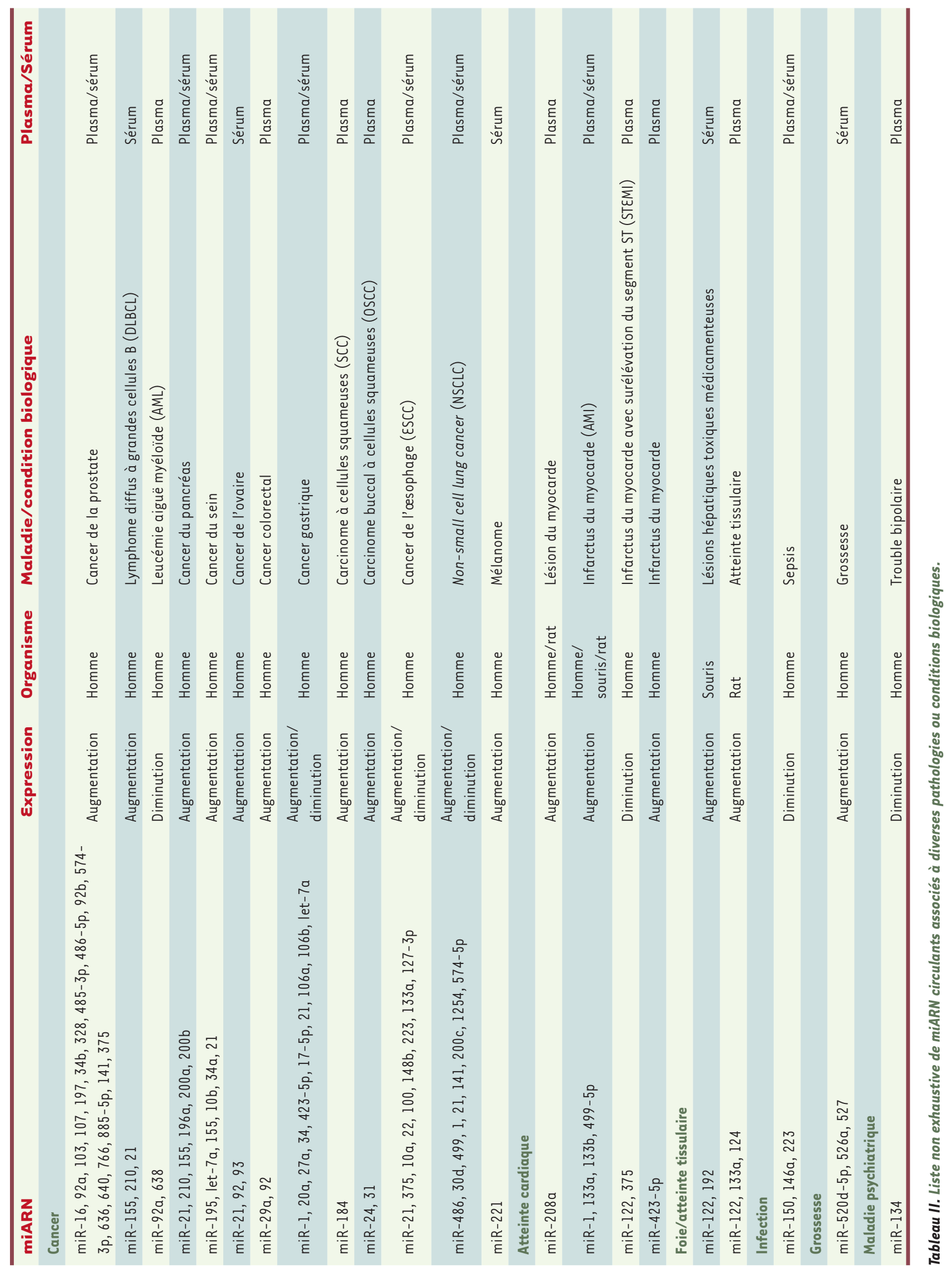




\section{Méthodologie pour le dosage des miARN circulants}

L'intérêt majeur de l'utilisation des miARN circulants comme biomarqueurs réside tout d'abord dans leur pertinence biologique, leur capacité à être quantifiés de manière fiable ainsi que leur présence dans des fluides biologiques accessibles (majoritairement le sang). Cependant, plusieurs considérations techniques doivent être prises en compte, car la reproductibilité des tests dépend d'une homogénéisation de la pratique qui n'est pas forcement présente dans la littérature. Tout d'abord, le mode de préparation des échantillons nécessite deux centrifugations successives: la première à faible vitesse $(1200 \mathrm{~g})$ pour se débarrasser des cellules et la suivante à une vitesse plus élevée (14000-16000 g) permettant d'éliminer les débrits cellulaires. Cette dernière étape a une importance majeure, car elle peut grandement interférer avec la détection de miARN d'intérêt. Par la suite, l'extraction des ARN doit être réalisée dans des conditions adaptées à la collecte de ces petits ARN, et doit tenir compte de la quantité de miRNA attendue. En effet, pour optimiser le rendement, l'ajout d'un agent co-précipitant peut être requis; de plus, il permet de limiter la perte de certaines populations de miARN, notamment ceux dont le contenu en GC (qui sont souvent perdus lors des extractions) est faible. Le choix de la méthode a elle aussi son importance. Il existe actuellement trois principales méthodes de dosage : la RT-PCR (reverse-transcriptase polymerase chain reaction) quantitative, le microarray et le séquençage haut-débit (NGS pour nextgeneration sequencing). Les deux dernières permettent une caractérisation globale des miARN et sont le plus souvent appliquées dans un but de recherche. Pour une approche ciblée analysant un nombre de miARN restreint, la RT-PCR s'avère plus adaptée car plus rapide et facilement transférable en clinique. La question des gènes de référence à utiliser pour normaliser les résultats est aussi un point important qui nécessite d'être défini au mieux pour chaque application. Le dosage de miARN circulants reste donc tout à fait possible pour une application de routine en clinique, dès l'établissement de procédures techniques standardisées.

non-small cell lung cancer (NSCLC) chez plus de six patients sur sept à partir d'une étude rétrospective, et cela 33 mois avant que le diagnostic officiel ne soit établi [33].

Les cancers du sein représentent $30 \%$ des cancers chez la femme et il y a un besoin important de biomarqueurs pour le diagnostic précoce. Le dépistage utilise actuellement la mammographie, qui reste coûteuse et difficile à systématiser. De plus, les marqueurs sanguins utilisés actuellement (le carcino-embryonic antigen [CEA] et le carcinoma antigen-15-3 [CA15-3]) manquent encore cruellement de précision. Une alternative nouvelle serait de doser le miR-195 dans le sang car son augmentation est précoce et survient chez les patientes porteuses de tumeurs de moins de deux $\mathrm{cm}$ de diamètre. De plus, le niveau de miR-195 dans le sang semble corrélé à la taille de la tumeur et retourne même à un niveau basal deux semaines après résection de la tumeur, indiquant le potentiel important de ce miR pour le suivi des patientes [34].

Dans le cancer colorectal (CRC) également, les marqueurs sanguins disponibles en clinique (CEA et CA19-9) restent insuffisants pour permettre un diagnostic précoce et fiable. Des résultats récents indiquent que le taux de miR-92 augmente très précocement dans le plasma des patients atteints d'un cancer colorectal, dès le stade 1 de la tumeur. Son dosage, seul ou en association avec d'autres miARN, comme miR-29a par exemple, pourrait venir compléter les tests sanguins actuels et améliorer la fiabilité du diagnostic [35]. Une telle démarche s'applique aussi au cancer du pancréas [39] dont la survie reste extrêmement faible à cinq ans, particulièrement si le diagnostic est tardif [39]. Une récente étude a mis en évidence une signature basée sur la combinaison de deux miARN (miR-16 et miR-196a) et du marqueur CA19-9, qui permet d'identifier avec une grande fiabilité la présence de tumeurs, y compris à des stades précoces [36, 39].

Cependant, les applications potentielles des miR circulants dépassent de loin le cadre de l'oncologie et touchent l'ensemble des pathologies humaines. Les maladies cardiovasculaires, elles aussi, pourraient tirer avantage de tests diagnostiques basés sur les miARN circulants. Ces derniers ont aussi leur place dans la détection des rejets de greffes, notamment dans le cas de greffes rénales. En effet, le taux de miR-204 dans les urines est diminué d'un facteur 300 chez les patients atteints de fibrose interstitielle et d'atrophie tubulaire du greffon rénal qui peuvent mener au rejet. De la même manière, le taux urinaire de miR-142-3p augmente parallèlement au rejet de la greffe, et ce avec une très bonne précision [37].

\section{Conclusion}

Grâce à leur stabilité et leur accessibilité, les miARN circulants représentent une nouvelle famille de biomarqueurs à très fort potentiel. Leur utilisation dépasse largement le champ de l'oncologie et s'adresse à un très large spectre de pathologies. Les besoins en biomarqueurs pertinents et peu invasifs sont multiples, allant du diagnostic précoce précédant l'apparition des premiers symptômes au suivi des patients, à la caractérisation de leur hétérogénéité face à la maladie et au traitement, constituant le concept de théranostic. La disponibilité de nombreuses platesformes de microarray capables de faire le typage presque exhaustif des 2578 miARN humains connus [10] à ce jour devrait accélérer encore la découverte de nouveaux biomarqueurs présents dans les fluides biologiques, ce qui renforcerait l'arsenal thérapeutique disponible pour les cliniciens. À l'ère de la médecine personnalisée et du théranostic, les miARN circulants représentent un nouveau défi plein de promesses. $\diamond$

\section{SUMMARY}

Circulating miRNAs as a new class of biomedical markers

Search for new biomarkers able to improve patient diagnosis has become a real challenge in the era of personalized medicine. Considering that early detection 
of cancer greatly increases the chances for successful treatment, the identification of accurate biomarkers is essential. In addition, the possibility to optimize patients' care and follow-up using "companion" diagnostic tests is emerging under the term of theranostics and should become a key step in future therapeutics. Since their discovery in serum and their close associations with pathological conditions, miRNA found in biological fluids represent a new promising class of potential biomarkers. First identified in cancer patients, possible applications are very broad and address the vast majority of human diseases. This review focuses on recent advances in our knowledge of circulating miRNA. Their production in cells, export in extra-cellular environment and presumed physiological functions are addressed. The review also emphasizes their potential applications in clinics as biomarkers or therapeutic agents. $\diamond$

\section{LIENS D’INTÉRÊT}

Les auteurs déclarent n'avoir aucun lien d'intérêt concernant les données publiées dans cet article.

\section{RéFÉRENCES}

1. Swarup V, Rajeswari MR. Circulating (cell-free) nucleic acids. A promising, non-invasive tool for early detection of several human diseases. FEBS Lett 2007 ; 581 : 795-9.

2. Gilad S, Meiri $\varepsilon$, Yogev Y, et al. Serum microRNAs are promising novel biomarkers. PLoS One 2008 ; $3: 3148$.

3. ENCODE project consortium. Identification and analysis of functional elements in $1 \%$ of the human genome by the ENCODE pilot project Nature 2007 ; 447 : 799-816.

4. Mattick JS, Makunin IV. Non-coding RNA. Hum Mol Genet 2006 ; 15 : R17-29.

5. Lee RC, Feinbaum RL, Ambros V. The C. elegans heterochronic gene lin- 4 encodes small RNAs with antisense complementarity to lin-14. Cell $1993 ; 75: 843-54$

6. Wightman B, Ha I, Ruvkun G. Posttranscriptional regulation of the heterochronic gene lin- 14 by lin-4 mediates temporal pattern formation in C. elegans. Cell $1993 ; 75: 855-62$.

7. Filipowicz W, Bhattacharyya SN, Sonenberg N. Mechanisms of post-transcriptional regulation by microRNAs: are the answers in sight? Nat Rev Genet 2008 ; 9 : 102-14.

8. Dunoyer $P$. La bataille du silence : mécanisme et inhibition du RNA silencing au cours des interactions plante/virus. Med Sci (Paris) 2009 ; 25 : 505-12.

9. Sacco L Da, Masotti A. Recent insights and novel bioinformatics tools to understand the role of microRNAs binding to 5' untranslated region. Int J Mol Sci 2012; $14: 480-95$.

10. Kozomara A, Griffiths-Jones S. miRBase: integrating microRNA annotation and deep-sequencing data. Nucleic Acids Res 2011 ; 39 : D152-7.

11. Friedman RC, Farh KK-H, Burge CB, et al. Most mammalian mRNAs are conserved targets of microRNAs. Genome Res $2009 ; 19: 92-105$.

12. Ventura A, Jacks T. MicroRNAs and cancer: short RNAs go a long way. Cell $2009 ; 136: 586-91$.

13. Zhang $Y$, Liu $D$, Chen $X$, et al. Secreted monocytic miR- 150 enhances targeted endothelial cell migration. Mol Cell $2010 ; 39$ : 133-44.

14. Hergenreider $\varepsilon$, Heydt $S$, Tréguer $K$, et al. Atheroprotective communication between endothelial cells and smooth muscle cells through miRNAs. Nat Cell Biol $2012 ; 14: 249-56$.

15. Mathivanan S, Fahner CJ, Reid GE, et al. ExoCarta 2012: database of exosomal proteins, RNA and lipids. Nucleic Acids Res $2012 ; 40$ : D1241-4.

16. Zernecke A, Bidzhekov K, Noels $H$, et al. Delivery of microRNA-126 by apoptotic bodies induces CXCL12-dependent vascular protection. Sci Signal $2009 ; 2$ : ra81.

17. Vickers KC, Palmisano BT, Shoucri BM, et al. MicroRNAs are transported in plasma and delivered to recipient cells by high-density lipoproteins. Nat Cell Biol 2011; 13:423-33.

18. Arroyo JD, Chevillet JR, Kroh EM, et al. Argonaute2 complexes carry a population of circulating microRNAs independent of vesicles in human plasma. Proc Natl Acad Sci USA 2011 ; 108 : 5003-8.
19. Turchinovich A, Weiz L, Langheinz A, et al. Characterization of extracellular circulating microRNA. Nucleic Acids Res 2011 ; 39 : 7223-33.

20. Russo F, Bella S Di, Nigita G, et al. miRandola: extracellular circulating microRNAs database. PLoS One $2012 ; 7$ : e47786.

21. Weber JA, Baxter DH, Zhang $S$, et al. The microRNA spectrum in 12 body fluids. Clin Chem $2010 ; 56: 1733-41$.

22. Laterza OF, Lim L, Garrett-Engele PW, et al. Plasma microRNAs as sensitive and specific biomarkers of tissue injury. Clin Chem 2009 ; 55 : 1977-83.

23. Montecalvo A, Larregina AT, Shufesky WJ, et al. Mechanism of transfer of functional microRNAs between mouse dendritic cells via exosomes. Blood $2012 ; 119: 756-66$

24. Tréguer $K$, Heydt $S$, Hergenreider $\varepsilon$. Protection des vaisseaux sanguins contre l'athérosclérose : le rôle des miARN sécrétés par l'endothélium. Med Sci (Paris) $2012 ; 28: 584-7$.

25. Kogure $T$, Lin W-L, Yan IK, et al. Inter-cellular nanovesicle mediated microRNA transfer: a mechanism of environmental modulation of hepatocellular cancer cell growth. Hepatology 2011 ; 54 : 1237-48.

26. Fabbri M, Paone A, Calore F, et al. MicroRNAs bind to Toll-like receptors to induce prometastatic inflammatory response. Proc Natl Acad Sci USA 2012 ; 109 : ع2110-6.

27. Lawrie CH, Gal S, Dunlop HM, et al. Detection of elevated levels of tumourassociated microRNAs in serum of patients with diffuse large B-cell lymphoma. BrJ Haematol 2008 ; 141 : 672-5.

28. Guire V De, Robitaille R, Tétreault N, et al. Circulating miRNAs as sensitive and specific biomarkers for the diagnosis and monitoring of human diseases: promises and challenges. Clin Biochem 2013; 46:846-60.

29. Corsten MF, Dennert R, Jochems S, et al. Circulating microRNA-208b and microRNA-499 reflect myocardial damage in cardiovascular disease. Circ Cardiovasc Genet $2010 ; 3: 499-506$.

30. Cermelli S, Ruggieri A, Marrero JA, et al. Circulating microRNAs in patients with chronic hepatitis $\mathrm{C}$ and non-alcoholic fatty liver disease. PLoS One $2011 ; 6$ : e23937.

31. Zampetaki A, Kiechl S, Drozdov I, et al. Plasma microRNA profiling reveals loss of endothelial MiR-126 and other microRNAs in type 2 diabetes. Circ Res $2010 ; 107: 810-7$.

32. Gilad S, Lithwick-Yanai G, Barshack I, et al. Classification of the four main types of lung cancer using a microRNA-based diagnostic assay. J Mol Diagn $2012 ; 14: 510-7$

33. Chen $\mathrm{X}, \mathrm{Hu} Z$, Wang $\mathrm{W}$, et al. Identification of ten serum microRNAs from a genome-wide serum microRNA expression profile as novel noninvasive biomarkers for nonsmall cell lung cancer diagnosis. Int J Cancer 2012 ; 130 : 1620-8.

34. Heneghan HM, Miller N, Kerin MJ. Circulating microRNAs: promising breast cancer biomarkers. Breast Cancer Res $2011 ; 13: 402$

35. Huang Z, Huang D, Ni S, et al. Plasma microRNAs are promising novel biomarkers for early detection of colorectal cancer. Int J Cancer $2010 ; 127$ : 118-26.

36. Liu J, Gao J, Du Y, et al. Combination of plasma microRNAs with serum CAl99 for early detection of pancreatic cancer. Int J Cancer 2012; $131: 683-91$.

37. Scian M, Maluf D, David K, et al. MicroRNA profiles in allograft tissues and paired urines associate with chronic allograft dysfunction with IF/TA. Am J Transplant $2011 ; 11: 2110-2$.

38. Hinault $C$, Dumortier 0 , Van Obberghen $\varepsilon$. MicroARN et diabète : petites structures, grands effets. Med Sci (Paris) $2013 ; 29$ : 785-90.

39. Bournet $B$, Dufresne $M$, Selves J, et al. Oncogène Kras et cancer du pancréas : trente ans plus tard. Med Sci (Paris) $2013 ; 29: 991-7$.

\section{TIRÉS À PART}

S. Baulande

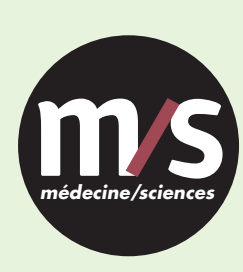

Tarifs d'abonnement $m / s-2014$

Abonnez-vous

à médecine/sciences
$>$ Grâce à $m / s$, vivez en direct les progrès des sciences biologiques et médicales

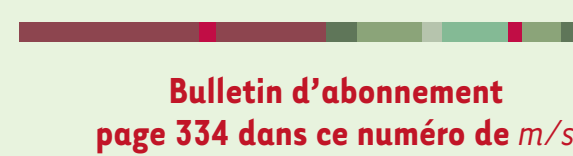

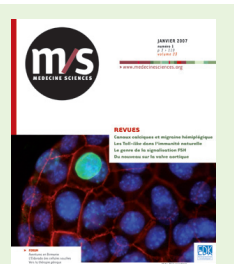

